\title{
Cognitive Behavioral Therapies for Insomnia
}

Editor

JASON C. ONG

\section{SLEEP MEDICINE CLINICS}

www.sleep.theclinics.com

Consulting Editor

TEOFILO LEE-CHIONG Jr

June 2019 • Volume 14 - Number 2 